\title{
Derivation of the System Equation for Null-Balanced Total-Power Radiometer System NCS1
}

\section{Sunchana P. Pucic}

National Institute of Standards and Technology, Boulder, CO 80303-3328

\begin{abstract}
A systcm equation of a recently devetoped null]-balanced, total-power radioncter system is rigorously derived. Delivered noise power and iemperature is related to availahle powcr (temperature) through an extcnston of the mismatch factor to broadband systems. The available power ratio $\alpha_{e}$, the available gain $G_{k}$ and the delivered power ratio (efficiency) $\eta_{1}$ are defincd. Properties of idealized, but in principle realizable components such as an infinitely directive isolator and a lossless matched waveguide-below-cutoff attenuator are used. A cascading technique is repeatediy applied to the fundamental noise
\end{abstract}

equation. Mathematically modeling the experimental procedure of sequentially attaching the two noise standards and the unknown suutce to the system input, we obtain the system of three equations that can be solved for the noise temperature of the unknown noise source.

Key words broadband; calibration; noise power, noise temperature; nullbalanced; system equation; total power radiometer section.

Accepted: October 12, 1993

\section{Introduction}

The system equation of a recently developed Noise Calibration System Model 1 (NCS1) is rigorously derived in this article. The NCS1, recently built at the National Institute of Standards and Technology, is used to measure thermal noise from coaxial noise sources. It consists of two noise standards and a null-balanced, total-power radiometer. The radiometer downconverts the amplified RF noise signal to a $30 \mathrm{MHz}$ IF frequency. A precision waveguide-below-cutoff (WBCO) attenuator is used to achieve a balanced operations mode. An unbased square law diode serves as a detector. Nulling and resolution control are implemented at the post-detection, DC stage. The system is described in more detail in the accompanying articles [1] and [2].

The noise power available from a noise source and measured by the NCS1 is calculated according to the system equation. This article presents a rigorous derivation of that equation. Initially, the equation is derived for an idealized, simplified system (the simplified equation). Modifications of the simplified system needed to actually implement the NCS1, and the resulting modifications of the simplified system equation, are described later. Deviations from ideal behavior are treated in [2].

\section{Derivation of the Simplified System Equation}

\subsection{Basic Considerations}

Several topics are briefly reviewed, in preparation for a derivation of the system equation.

2.1.1 Symbols In the following text, terms a noise source and a noise generator are used interchangeably; the subscript $g$ designates a general, unspecific origin of the noise signal $\left(\mathrm{e}_{\mathrm{g}}, P_{\mathrm{g}}\right)$. The specific noise sources are identified with subscripts $s$ for a noise standard (e.g, $P_{s}$ ) and $x$ for the 
unknown (e.g., $P_{x}$ ). Terms the unknown noise source and the DUT (the device under test) are used interchangeably. The subscript a stands for the ambient temperature (as in $P_{2}$ ).

Capital letter subscripts designate parts of the radiometer. The capital $A$ stands for the attenua* tor, $G$ for the amplifier, I for the isolator, and $\mathrm{SW}$ for the input switch.

For reflection coefficients within the radiometer, $\bar{\Gamma}$ signifies a reflection coefficient looking backward, in the direction opposite to the net power flow.

The subscript $i$ in many of the quantities signifies that quantity's dependency on the existing input conditions. Initially, $i$ stands for 1,2 , or $x$, that is, the conditions of having the noise standard 1 , the noise standard 2, or the unknown noise source $x$ attached to the input. Later on, $i$ stands for $s, a$, or $x$, that is, the conditions of having the nonambient noise standard, the ambient noise standard, or the unknown noise source $x$ attached to the input.

2.1.2 The Measurement Quantity The quantity measured by the NCS1 is the electromagnetic noise power $P$, in watts; however, it is the noise temperature $T$, in kelvins $(\mathrm{K})_{3}$ that is customarily reported. Neglecting quantum effects, the two quantities are proportional to each other according to the well known relationship

$$
P=k B T \text {, }
$$

where $k$ is the Boltzmann constant $=1.38 \cdot 10^{-23}$ $J / K$, and $B$ is the system noise bandwidth in hertz.

The quantities $P$ and $T$ are the available noise power and the available temperature: the power or temperature that would be delivered by a source to a load, such as a power meter, under the ideal conditions of a complex conjugate match.

In contrast, the delivered power $\boldsymbol{P}_{\mathrm{det}}$ (or temperature $T_{\text {dei }}$ ) is the power (temperature) that is actually delivered to a (generally mismatched) load.

2.1.3 The Mismatch Factor The available and the delivered powers (temperatures) are related by

$$
\boldsymbol{P}_{\mathrm{del}}=M P_{\text {, }}
$$

and

$$
\tau_{\text {del }}=M T
$$

where $M$ is the mismatch factor, a real number between 0 (total mismatch), and 1 (perfect match). The mismatch factor is a function of the reflection coefficients of the source and the load. Power delivered to a load from sources having the same available power, but different reflection coeffcients, is therefore variable and dependent on the input conditions.

In broadband systems of bandwidth $B$, the mismatch factor, if used as in Egs. (2) and (3) is strictly defined as

$$
M=\int_{a} M(f) g \mathrm{~d} f
$$

where $g$ is the transfer function of the radiometer, and $f$ is the frequency.

In principle, the mismatch factor can be evaluated according to Eq. (4). However, as long as the elements constituting $M$ do not change appreciably across the measurement band, the use of a constant mismatch factor evaluated conveniently at mid-band produces negligible errors. Proper engineering design must assure a compliance with Eq. (4) and the error analysis must evaluate a deviation from the ideal conditions $[3,4]$.

2.1.4 Passive Two-Ports If a noise generator with the available noise temperature $T_{\mathrm{B}}$ is connected to the input of a passive, linear two-port held at the temperature $T$, the available temperature $T_{\text {ous }}$ at the output of the two-port can be calculated from fundamental thermodynamic principles. It is given by $[5,3]$ by

$$
T_{\text {oul }}=T_{\mathrm{q}} \alpha_{\mathrm{s}}+T\left(1-\alpha_{\mathrm{g}}\right) \text {. }
$$

The quantity $a_{\mathrm{f}}$ is a ratio of the avajlable power at the output of the two-port to the available power at the input of the two-port; $\alpha_{\mathrm{g}} \equiv P_{\text {out }} / P_{\mathrm{in}}$. The quantity $\alpha_{\mathrm{g}}$ is an in situ quantity; it depends not only on the parameters of the two-port, but also on those of the two-port's environment [5]. Specifically, it depends on the reflection coefficient looking into the network preceding the two-port, that is, the (possibly equivalent in a Thevenin sense) generator. This dependence on the input conditions is indicated by the subscript $\mathrm{g}$. The quantity $\alpha_{k}$ characterizes the Jossiness of the two-port and has a value between 0 (an infinitely lossy two-port) and 1 (a lossless two-port). A full definition of $\alpha_{\mathrm{g}}$, applied to the specific passive two-port within its environment, is given in Sec. 2.3.

The first term $T_{k} \alpha_{B}$ in Eq. (5) describes how the input noise signal is modified by a passage through the two-port. The second term $T\left(1-\alpha_{4}\right)$ describes the noise contribution of the two-port itself.

2.1.5 Active Two-Ports If a nolse source with the available noise temperature $T_{g}$ is connected to 
the input of an active linear two-port such as an amplifier, the available temperature $T_{\text {out }}$ at the output of the two-port is given by

$$
T_{\text {cut }}=G_{\xi}\left(T_{\xi}+T_{e t}\right)
$$

The quantity $G_{t}$ is the available gain of the active two-port. Similar to $\alpha_{b}$, it is a ratio of the available power at the output of the two-port to the available power at the input of the two-port, $G_{g} \equiv P_{\text {out }} / P_{\text {in }}$ As an in situ quantity, it is dependent on the input conditions, as emphasized by the subscript g. A full definition, applied to the specific active two-port within its environment, is given in Sec. 2.3.

The quantity $T_{e q}$ in $\mathrm{Eq}$. (6) accounts for the noise contribution of the active two-port, a function of the circuit design and only marginally dependent on the physical temperature. The noise that is generated by the active two-port and is avalable at its output, is referred to its input as the effective input noise temperature $T_{c \mathrm{p}}$. The subscript $\mathrm{g}$ emphasizes its dependency on the reflective properties of the (possibly equivalent) generator (Sec. 2.3).

2.1.6 A Cascade From the definitions of $\alpha$ and $G$ it follows that for a cascaded chain of $n$ elements, the overall $\alpha_{c s c}=\alpha_{1} \alpha_{2} \ldots \alpha_{n}$, and $G_{\text {asc }}=$ $G_{1} G_{2}, \ldots G_{n}$

\subsection{Basic Assumptions}

The derivation of the system equation is based on repeated applications of Eqs. (5) and (6) to the elements of the cascaded chain of the radiometer. The conditions assumed to hold are:

- Steady state

- Single-mode propagation in the transmission lines

- Lossless transmission lines and ideal connectors

- Linear elements throughout, except for the square-law power detector

- Broadband components across the bandwidth $B$

- Thermal noise (i.e., noise having a Gaussian amplitude distribution and a flat power spectrum across the noise bandwidth $B$ ) generated by the noise sources

- All passive components in the radiometer at the ambient temperature $T$.

- A linear WBCO attenuator in its dial settings.

\subsection{The Simplified Radiometer System}

For the purpose of deriving the system equation, the radiometer is reduced to essential components: an isolator, an RF amplifier, a WBCO attenuator with its matching pads (Sec. 2.3.2 and [6]), and a receiver containing a square law detector (Fig. 1). The lossiness of the input section of the radiometer is combined with the lossiness of the isolator. The isolator itself is initially treated as a simple lossy, reciprocal two-port, and later (Sec. 2.3.2) it assumes the nonreciprocal properties. The amplifier is the element assumed to define the system bandwidth.

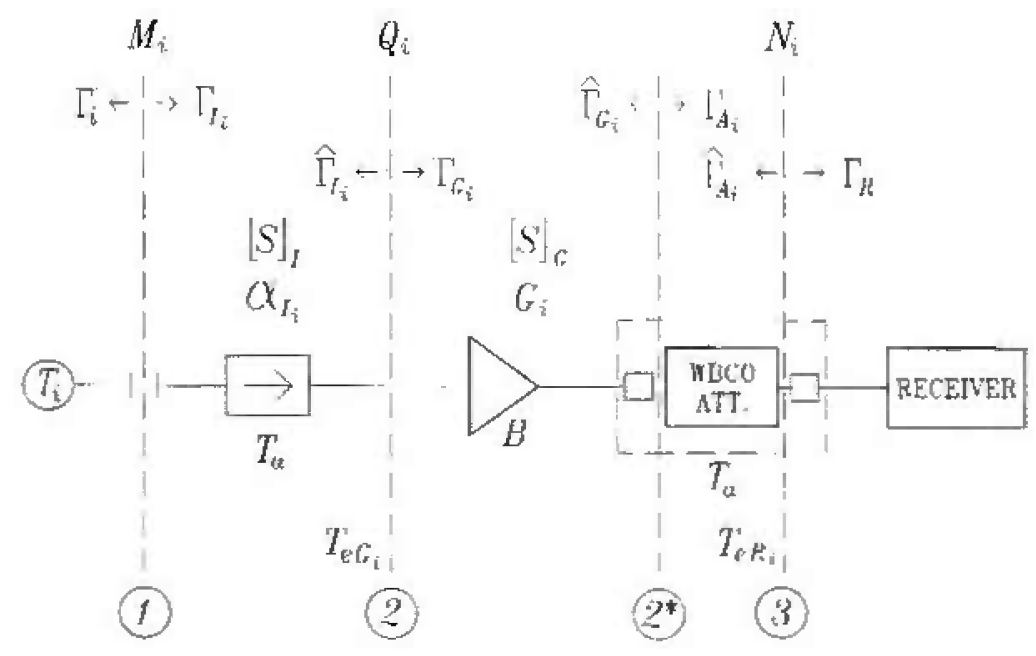

Fig. 1. Simplified system block diagrami. 
The two noise standards are characterized by their available noise temperatures $T_{1}$ and $T_{2}$ and their reflection coefficients $\Gamma_{1}$ and $\Gamma_{2}$. The DUT has a known (measured) reflection coefficient $\Gamma_{\mathrm{x}}$. Its available temperature is the quantity under test.

The radiometer input signal is the broadband noise generated by the three noise sources: the two noise standards and the DUT. The three sources are sequentially attached to the radiometer input port and the noise power is adjusted by the attenuator so that the receiver measures the same power in all three cases.

The radiometer reflection coefficient (at the input plane 1 in Fig. 1) is $\Gamma_{\mathrm{I}_{\mathrm{j}}}$. In general, it varies with the attenuator setting, since it depends on the (variable) $\Gamma_{A_{f}}$, according to [7]:

$$
\Gamma_{\mathbf{t}_{t}}=S_{1 \mathrm{1}_{1}}+\frac{S_{\mathrm{Iz}_{1}} S_{2 \mathrm{~h}_{1}} \Gamma_{\mathrm{G}_{j}}}{1-S_{2 \mathrm{z}_{1}} \Gamma_{\mathrm{G}_{j}}}
$$

while the term $\Gamma_{\mathrm{C}_{j}}$ is, in turn, given by

$$
\Gamma_{\mathrm{G}_{i}}=S_{\mathrm{II}_{\mathrm{G}}}+\frac{S_{12_{\mathrm{g}}} S_{21_{\mathrm{G}}} \Gamma_{\mathrm{A}_{i}}}{1-S_{2 \mathrm{Z}_{G}} \Gamma_{\mathrm{A}_{i}}}
$$

In the preceding two equations, the $S$-parameters $[S]_{1}$ characterize the isolator, while $[S]_{\mathrm{c}}$ pertain to a cascade of the amplifier and the left-sided matching pad of the WBCO attenuator.

The effective input noise temperature of the amplifier $T_{\mathrm{e}} \mathrm{G}_{t}$ varies with the input conditions. The effective input noise temperature of the receiver $T_{\mathrm{eR}_{i}}$ varies with both the input conditions and the attenuator settings.

2.3.1 Equations Modeling the Measurement Procedure The measurement procedure consists of attaching the two noise standards and the DUT to the radiometer input port in sequence, and adjusting the attenuator settings until a balanced condition is achieved.

With the first noise standard (with available noise power $k B T_{1}$ and reflection coefficient $J_{1}$ ) attached to the radiometer input, the receiver detects a certain amount of delivered power $P_{\text {dcl: }}$ :

$$
\begin{gathered}
k B\left\{\left[T_{\mathrm{l}} \alpha_{\mathrm{I}_{\perp}}+T_{\mathrm{I}}\left(1-\alpha_{\mathrm{I}_{\mathrm{l}}}\right)+T_{\left.\mathrm{eG}_{\mathrm{l}}\right]}\right] G_{\mathrm{l}} \cdot \alpha_{\mathrm{A}_{\mathrm{l}}}+T_{\mathrm{a}}\left(1-\alpha_{\mathrm{A}_{\mathrm{l}}}\right)+\right. \\
\left.T_{\mathrm{eR}}\right\} \cdot N_{1}=P_{\text {del }} .
\end{gathered}
$$

The term $G_{1}$ (and likewise $G_{2}$ and $G_{x}$ in the following equations) refers to the available gain of the amplifier/left-matching-pad cascade (Sec. 2.1.6).

As the second noise standard (with available noise power $k B T_{2}$ and reflection coefficient $\Gamma_{2}$ ) is attached to the radiometer input, the attenuator is adjusted so that the receiver detects the same delivered power $P_{\text {del }}$ as previously:

$$
\begin{gathered}
k B\left\{\left[T_{2} \alpha_{1_{z}}+T_{1}\left(1-\alpha_{\mathrm{I}_{2}}\right)+T_{\mathrm{eG}}\right] G_{2} \cdot \alpha_{\mathrm{A}_{7}}+T_{\mathrm{z}}\left(1-\alpha_{\mathrm{A}_{2}}\right)+\right. \\
\left.T_{\mathrm{eR} \mathrm{R}_{2}}\right\} \cdot N_{2}=P_{\text {del. }}
\end{gathered}
$$

Finally, as the DUT (with avaitable noise power $k B T_{x}$ and reflection coefficient $\Gamma_{x}$ ) is attached to the radiometer input, the attenuator is again adjusted so that the receiver detects the same delivered power $P_{d \in l}$ as in the previous two cases:

$$
\begin{gathered}
k B\left\{\left[T_{x} \alpha_{l_{x}}+T_{x}\left(1-\omega_{x}\right)+T_{e G_{r}}\right] G_{x}-\alpha_{A_{x}}+\right. \\
\left.T_{a}\left(1-\alpha_{A_{x}}\right)+T_{e R_{y}}\right\}+N_{x}=P_{\text {dels }}
\end{gathered}
$$

where $k$ is the Boltzmann constant, $B$ is the limiting system bandwidth, $T_{i}(i=1,2, x)$ is the available noise temperature of the noise sources attached to the input, and $\alpha_{i}$ and $\alpha_{\mathrm{A}_{\mathrm{i}}}$ are the available power ratios (Sec. 2.1), in siru quantities. They are defined for the specific passive two-ports within their respective environments [5], as

$$
\alpha_{i_{i}}=\frac{\left(1-\left|\Gamma_{\mathrm{i}}\right|^{2}\right\rangle \cdot\left|S_{21,}\right|^{2}}{\left.\left(1-\left|\bar{\Gamma}_{\mathrm{t}_{i}}\right|^{2}\right)\right] 1-\left.S_{11_{\mathrm{i}}} \Gamma_{\mathrm{i}}\right|^{2}}
$$

for the isolator, and

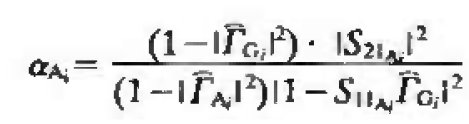

for the altenuator.

In Eqs. (12) and (13) $S_{21_{1}}, S_{11_{1}}$ and $S_{2 i_{A_{i}}}, S_{1 t_{A_{i}}}$ are the $S$-parameters of the isolator and the attenuator. The $S$-parameters of the attenuator vary with the attenuator setting.

The terms $\Gamma_{i}, \widehat{\Gamma}_{I_{i}}, \overleftarrow{\Gamma}_{\mathrm{G}_{i}}$, and $\widehat{\Gamma}_{\mathrm{A}}$ in the same equations are the reflection coefficients shown in Fig. 1. The fact that $\alpha_{]_{i}}$ and $\alpha_{A_{j}}$ have reflection coefficients looking either directly into the sources $\left(\Gamma_{i}\right)$, or indirectly into the sources through one or more twoports $\left(\widehat{I}_{1}, \widehat{\Gamma}_{\mathrm{G}_{i}}\right.$, or $\left.\bar{\Gamma}_{\Lambda_{i}}\right)$, explicitly shows their dependency on the input conditions.

$T_{\mathrm{eq}}$ and $T_{\mathrm{eR}_{i}}$ are the effective input noise temperatures, in kelvins, of the amplifier and the receiver. The effective input noise temperature $T_{\mathrm{r}} \mathrm{G}_{i}$ of the $\mathrm{RF}$ amplifier depends on the input conditions, i.e., on the reflection coefficients of the three noise sources $\Gamma_{\text {. }}$. The effective input noise temperature of the receiver $T_{t \mathrm{~B}_{i}}$ depends on the reflection coefficients of the three noise sources, as well as on the 
reflection coefficient looking backward into the attenuator at its three different settings $\bar{\Gamma}_{A_{i}}$.

The available gain of the amplifier/left-matchingpad combination $G_{i}$, in situ quantity, is given by

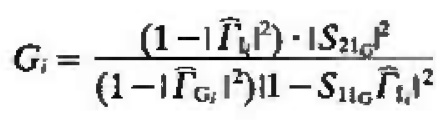

The terms $\widehat{\Gamma}_{\mathrm{f}}$ and $\widehat{\Gamma}_{\mathbf{G}_{i}}$ in Eq. (14) explicitly show the dependency of the available gain $G_{i}$ on the input conditions. In the case of $\widehat{\Gamma}_{l_{i}}$, this dependency is direct:

$$
\bar{\Gamma}_{1,}=S_{22_{1}}+\frac{S_{13_{1}} S_{21} \Gamma_{1}}{1-S_{11} \Gamma_{i}}
$$

In the case of $\bar{\Gamma}_{\mathrm{G}_{i}}$, the dependency is indirect, through the $\bar{\Gamma}_{\mathrm{L}}$ dependency on the input conditions:

$$
\widehat{\Gamma}_{\mathrm{C}_{i}}=S_{2_{G}}+\frac{S_{12_{G}} S_{2 t_{G}} \widehat{\Gamma}_{I_{F}}}{1-S_{\sharp 1_{G}} \widehat{\Gamma}_{\mathrm{I}_{i}}}
$$

A mismatch factor $N_{i}$ is defined at the plane 3 in Fig. 1 as

$$
N_{i}=\frac{\left(1-\left|\bar{\Gamma}_{\mathrm{A}_{i}}\right|^{2}\right)\left(1-\left|\Gamma_{\mathrm{R}}\right|^{2}\right)}{\left|1-\bar{\Gamma}_{\mathrm{A}_{l}} \Gamma_{\mathrm{R}}\right|^{2}}
$$

where $\Gamma_{\mathrm{R}}$ is the reflection coefficient looking into the receiver, and $\vec{\Gamma}_{A}$ is the reflection coefficientlooking backward into the attenuator, defined as

$$
\widehat{\Gamma}_{A_{i}}=S_{2 z_{A_{i}}}+\frac{S_{12_{A i}} S_{21_{k},} \widehat{\Gamma}_{\mathrm{G}_{i}}}{1-S_{1 I_{A_{i}}}}
$$

2.3.2 Idealized Elements Assumptions Equations (9)-(11) simplify considerably under the following assumptions:

- Infinitely directive $\left(S_{12_{1}}=0\right)$, but not necessarily lossless or matched isolator

- Large gain preceding the attenuator

- Lossless WBCO attenuator $\left(\alpha_{\Lambda}=1\right)$

The first assumption results in the following simplifications:

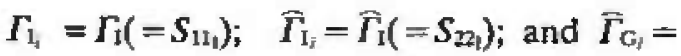

$$
\begin{aligned}
& \bar{\Gamma}_{\mathrm{G}}\left(=S_{2 z_{G}}\right) \\
& G_{i}=G \\
& T_{\mathrm{eG}}=T_{\mathrm{eG} ;} ; T_{\mathrm{eR} i} \neq f\left\{\Gamma_{i}\right\} .
\end{aligned}
$$

Because of the second assumption, the temperature of the input noise signal, once it reaches the input of the receiver, is much higher than the receiver effective input noise temperature $T_{\mathrm{eR}}$. Consequently, the variability of the receiver effective input noise temperature, oue to changes in the reflection coefficient of the receiver equivalent source $\Gamma_{A}$ (which in turn are related to different attenuator settings), becomes negligible: $T_{\mathrm{eR}_{i}}$ becomes the constant $T_{\mathrm{er}}$.

The implications of the third assumption are discussed next. An ideal WBCO attenuator is inherently a lossless device, because the attenuation is achieved by the input signal propagating under the below-cutoff conditions. The input signal is therefore partially reflected and partially transmitted; no component of the input signal is absorbed, so there are no losses. (Small losses within the imperfect metal walls of the implemented attenuator can be neglected in this derivation.) The term $\alpha_{A_{i}}$ becomes 1 , and consequently its noise contribution $T_{\mathbf{s}}\left(1-\alpha_{\mathrm{A}_{i}}\right)$ vanishes.

The concept of attenuation is valid only in a nonreflecting environment [7]. The WBCO attenuator has a pair of (lossy) matching pads that are placed at the input and output ports to fulfill the requirement. The two reference planes (planes $2^{*}$ and 3 in Fig. 1) are positioned between the WBCO attenaator proper and the matching pads, assuring a good bilateral match, while maintaining the lossless chasacter of the WBCO attenuator proper.

The mismatch factor $N_{i}$ depends on $\Gamma_{\mathrm{R}}$ and $\bar{\Gamma}_{A_{i}}$ [Eq. (17)]. The attenuator padding assures $\Gamma_{\mathrm{R}}$ $=\Gamma_{G}=0$. The condition $\Gamma_{\mathrm{R}}=0$ reduces the expression for the mismatch factor $N_{i}$ to $\left(1-\left|\widehat{\Gamma}_{A_{i}}\right|^{2}\right)$. Under the assumption that $\Gamma_{\mathrm{G}}=0$, the expression for $\bar{\Gamma}_{A_{l}}$, in turn, reduces to $S_{22_{A_{i}}}$. The WBCO attenuator, postulated to be a lossless device, must satisfy the losslessness condition $\left|S_{22_{A}}\right|^{2}=\left(1-\left|S_{21_{A}}\right|^{2}\right)$ [7] Under the assumptions that the attenuator is lossless and matched, the expression for the mismatch factor $N_{i}$ is drastically simplified to $N_{i}=\mid S_{\left.2 I_{A i}\right|^{2}}$.

With all of the above simplifications in mind, the initial set of three Eqs. (9)-(11) now become

$$
k B\left\{\left\{T_{1} a_{1_{1}}+T_{\mathrm{s}}\left(1-a_{\mathrm{T}_{\mathrm{s}}}\right)+T_{\mathrm{eG}}\right] G+T_{\mathrm{tR}}\right\}\left|S_{2 \mathrm{~A}_{\mathrm{A}_{1}}}\right|^{2}=P_{\mathrm{det}},
$$

$$
k B\left\{\left[T_{2} \alpha_{\mathrm{I}_{2}}+T_{\mathrm{2}}\left(1-\alpha_{\mathrm{T}_{2}}\right)+T_{\mathrm{eG}}\right] G+T_{\mathrm{eR}}\right\}\left|S_{2 \mathrm{~A}_{\mathrm{A}}}\right|^{2}=P_{\mathrm{del}}
$$

$$
k B\left\{\left[T_{k} \alpha_{\mathrm{l}_{x}}+T_{\mathrm{a}}\left(1-\alpha_{\mathrm{I}_{\mathrm{s}}}\right)+T_{\mathrm{EG}}\right] G+T_{\mathrm{tR}}\right\}\left|S_{21_{\mathrm{A}_{\mathrm{A}}}}\right|^{2}=P_{\mathrm{dkl} \mid}
$$

